Special issue of the 3rd International Conference on Computational and Experimental Science and Engineering (ICCESEN 2016)

\title{
Electronic System and Software Architecture of Modular Reconfigurable Robot Module OMNIMO
}

\author{
A. KILIÇ* AND S. KAPUCU \\ Gaziantep University, Mechanical Engineering Department, Gaziantep, Turkey
}

\begin{abstract}
A robot is an electromechanical system, which is guided by software via electronic circuitry. In the absence of electronic systems, there is no way to make a connection between software and mechanical components. This means that all robotic systems require mechanical systems, electronic systems and software. OMNIMO, as a modular reconfigurable robot, has sophisticated electronic systems and software. Electronic system of OMNIMO includes controllers, actuators, transducers, communication units, regulators, batteries, user interface units and complementary components. In addition, OMNIMO is controlled by four different layers of software. In this paper, electronic hardware details, system integration and control software architecture of modular reconfigurable robot module OMNIMO are presented. In addition, adaptation of components and communication protocol details of hardware are given.
\end{abstract}

DOI: 10.12693/APhysPolA.132.913

PACS/topics: 07.07.Tw, 45.40.Ln, 87.85.St

\section{Introduction}

Unlike the conventional robotic systems, modular reconfigurable robots combine the properties and capabilities of multi robot systems to accomplish the objectives, which are on the further side of the abilities of a single robot system. As expected, these robots are more complex than standard robots, concerning their design, controlling electronics and software.

A modular reconfigurable robot can be thought as a universal robot or a general-purpose robot. Potentially, they can be used in space explorations to dynamic furniture applications. In addition, a modular reconfigurable robot is capable of simulating any other physical robot. This capability arises from the facts that;

- These robots are composed of robotic modules.

- Their capability of modularity and redundant mobility, allows the robots to construct the shape of any other robot.

- They can connect with each other in various different forms in order to make two and/or three dimensional geometries.

These abilities make it possible for modular reconfigurable robots to adapt and optimize their shape for any new and unpredictable duty. For instance, a modular reconfigurable robot can change into the shape of a wheeled robot, to quickly travel on smooth roads, and then become a snake to explore a narrow pipe or cave, it can be a legged robot to carry load in rough terrain. Moreover, if it comes across a hill or mountain, it can become

*corresponding author; e-mail: akilic@gantep.edu.tr a climbing robot, and finally, it can be used as a structural element, such as table or wall. By using OMNIMO, so many single module and multi module robot alternatives can be constructed [1].

By this vision, a modular reconfigurable robot module OMNIMO [OMNIdirectional MOdular robot] is developed. OMNIMO has its own locomotion and navigation systems and it can accomplish some simple duties. For complex tasks suitable robot type can be constructed by using multi modules. Moreover, OMNIMO is a homogeneous type, hybrid, deterministic modular reconfigurable robot module. It has five degrees of freedom, four revolute and one prismatic. Degrees of freedom of the robot can be used as fixed, free and actuated. A photograph of OMNIMO can be seen in Fig. 1.

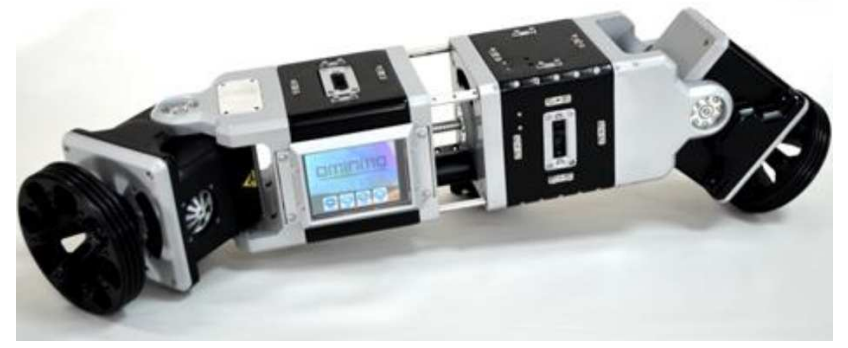

Fig. 1. Photograph of OMNIMO.

Up to now, more than seventy different modular reconfigurable robot systems were developed. There are many various forms, from one degree of freedom to six degrees of freedoms. In this part, a selection of modular robots is surveyed and analyzed, with a focus on most important features of modular robots, that are number of DoF (degrees of freedom), DoF types, developers, classification and production date of modular robots. In Table I, reader can find the robot modules in historical order. 
After inspecting modular robot modules and studies, there is limited information about electronic systems, integration, used components and software architecture.
Thus, in this paper, electronic systems and software architecture of a new modular reconfigurable robot module, which is called OMNIMO, are presented.

TABLE I

List of modular reconfigurable robotic systems.

\begin{tabular}{c|c|c|c|c|c|c|c}
\hline \hline Robot & DoF & DoF Type & Dimension & Architecture & Year & Developers & Reference \\
\hline CEBOT & 2 & $2 \mathrm{R}$ & Various & Mobile & 1988 & Fukuda et al. & {$[2,3]$} \\
PolyPod & 2 & $2 \mathrm{R}$ & 3D & Chain & 1993 & Mark Yim & {$[4]$} \\
Metamorphic & 6 & $6 \mathrm{R}$ & 2D & Lattice & 1993 & Chirikjian G.S. & {$[5]$} \\
CONRO & 2 & $2 \mathrm{R}$ & 3D & Chain & 1998 & Castano et al. & {$[6]$} \\
PolyBot & 1 & $1 \mathrm{R}$ & 3D & Chain & 1998 & Yim et al & {$[7]$} \\
3D Unit & 6 & $6 \mathrm{R}$ & 3D & Lattice & 1998 & Murata et al. & {$[8]$} \\
Molecule & 4 & $4 \mathrm{R}$ & 3D & Lattice & 1998 & Kotay et al. & {$[9]$} \\
Telecube & 6 & $6 \mathrm{P}$ & 3D & Lattice & 1998 & Suh et al. & {$[10]$} \\
M-TRAN & 2 & 2R & 3D & Hybrid & 1998 & Murata et al. & {$[11]$} \\
Crystalline & 1 & 1P & 2D & Lattice & 1999 & Rus and Vona & {$[12]$} \\
I-Cubes & 3 & $3 \mathrm{R}$ & 3D & Lattice & 1999 & Unsal et al. & {$[13]$} \\
M-TRAN II & 2 & 2R & 3D & Hybrid & 2002 & Murata et al. & {$[14]$} \\
Swarm-bot & 3 & 3R & 2D & Mobile & 2003 & Mondada et al. & {$[15]$} \\
ATRON & 1 & 1R & 3D & Hybrid & 2003 & Ostergard et al. & {$[16]$} \\
Catoms & 0 & - & 2D & Lattice & 2005 & Goldstein et al. & {$[17]$} \\
Molecube & 1 & 1R & 3D & Hybrid & 2005 & Zykov et al. & {$[18]$} \\
M-TRAN III & 2 & 2R & 3D & Hybrid & 2005 & Kurokawa et al. & {$[19]$} \\
SuperBot & 3 & 3R & 3D & Hybrid & 2005 & Salemi et al. & {$[20]$} \\
YaMoR & 1 & 1R & 2D & Chain & 2005 & Moeckel et al. & {$[21]$} \\
Y1 Modules & 1 & 1R & 2D & Chain & 2006 & Gonzalez et al. & {$[22]$} \\
Miche & 0 & - & 3D & Lattice & 2007 & Rus et al & {$[23]$} \\
Roombots & 3 & 3R & 3D & Hybrid & 2008 & Sproewitz et al. & {$[24]$} \\
Sambot & 3 & 3R & 3D & Hybrid & 2010 & Wei et al. & {$[25]$} \\
Smores & 4 & $4 \mathrm{R}$ & 3D & Hybrid & 2012 & Davey et al. & {$[26]$}
\end{tabular}

\section{Electronic system of OMNIMO}

In the hardware part, in order to get maximum performance from a robot, electronic and mechanical systems should be accurate and robust. Development of modular robot module requires sophisticated mechanical capabilities [27]. For this reason, when selecting electronics components, all the parts must be durable and compatible with each other. In addition, before selecting electronic components, general framework of software of the robot should be created and mechanical limitations should be identified. Software size and computation load should be roughly estimated, to select the correct controller. In this part of study, electronic hardware details of OMNIMO are presented. In addition, communication protocol details of parts are given.

\subsection{Controller}

A controller in a robot plays the role of the brain. Therefore, every mechatronic and robotic system needs a controller. Robot controllers provide an interface between inputs and outputs in a robot. These are programmable microcontrollers, microprocessors and computers that enable robot to sense and act in its working environment. In other words, robot controller is an electronic and programmable hardware and it is a connection between hardware and software. Generally, the robot controller receives signals from the operator interface or from sensors. According to the developed software, robot controller combines the measured sensor data and predefined tasks. The electronic controller makes the robot come to life.

In this robotic project, Dynamixel robotic servo has been used as an actuator. Dynamixel can be controlled using specific controllers and computers. To control Dynamixel, communication can be established by a proper protocol. Dynamixel uses the half-duplex universal asynchronous serial communication protocol (UART) with 8 bit, 1 stop bit, and none parity. The controller of the robot and Dynamixel communicate with each other by sending and receiving data packets. There are two types of packets:

- Instruction packet includes signals sent from the main controller to Dynamixel,

- Status packet includes the Dynamixel responses sent to main controller as a feedback.

Details of Dynamixel robotic servomotors can be found in $[28]$. 
In this research, each reconfigurable robotic module has five actuated DoF. It contains special sensors, such as gyroscope and distance measurement sensors. For the integration of the software, sensory equipment, communication elements and actuators, a special controller is needed. The motivation to build OMNIMO was to develop an open source modular robotic platform. For this reason Arduino MEGA 2560 has been used as the main controller. It can be easily obtained and it has high capacity.

Arduino MEGA 2560 is a control module type controller. It contains ATMega2560 microcontroller. ATMega2560 is a low power, high-performance, Atmel 8-bit AVR RISC-based microcontroller [29].

\subsection{Actuators}

A robot is equipped with the actuators in order to create motion. Thus motor and drives are called actuators. In our robotic project, Robotis brand Dynamixel robotic smart servo is selected, due to its robustness, high torque output, and high controllability. The Dynamixel servo modules provide an interface between controller and motors. The Dynamixel servos are smart and modular actuators that contain high quality precise DC motor, low backlash gearbox, and a control circuitry with networking functionality, all in a single housing. That is to say, they are high performance actuators for robots, fully integrated with feedback, networking, and programmability. In this study, due to the high torque requirement, Dynamixel EX 106+ will be used.

\subsection{Transducers}

Transducer is a device that measures some type of physical change in the environment. The changes could include motion, heat, pressure, force, light or any one of a great number of other environmental phenomena. The output of sensory systems is generally an analog or digital signal, that is converted and amplified to be understandable for the microcontroller, for reading or further processing. In OMNIMO project, nine different sensors are used. They are;

- 3 axis accelerometer

- 3 axis gyroscope

- 3 axis magnetometer

- Magnetic encoder

- Current sensor

- Voltage sensor

- Temperature sensor

- Ultrasonic distance sensor

- Limit switch
Accelerometers are devices that measure how fast something is speeding up or slowing down. Accelerometers are used to sense both dynamic and static (gravity) acceleration.

Gyroscopes are devices that measure angular velocity; how fast something is spinning about an axis.

Magnetometers or digital compasses are commonly used for measuring heading direction, using the magnetic field of Earth.

Accelerometers, gyroscopes, and magnetometers are very useful, but alone they do not give quite enough information, to be able to calculate orientation, position, and velocity. For that reason, in OMNIMO project IMU (inertial measurement unit) is used. An inertial measurement unit includes three different sensors. It uses accelerometer to detect the current rate of acceleration and detects changes in angular velocity using gyroscopes and uses magnetometer, in order to measure bodysurrounding magnetic field. However, IMU is used for calculating robot orientation. In this study, a robust orientation angles calculating software was developed by using Kalman filter and sensor fusion algorithm. Orientation angles, which are roll, pitch, and yaw, can be seen in Fig. 2.

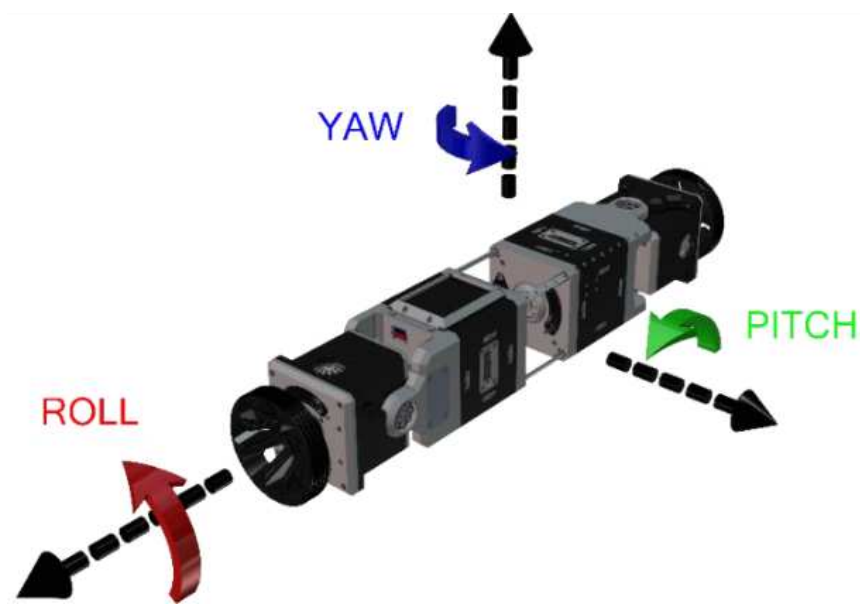

Fig. 2. Orientation angles of robot module.

An encoder is a sensor that is used to measure position, velocity, and acceleration of the motor. It is embedded in the servo motor. By using this encoder position, velocity, and acceleration of each axis can be measured.

A current sensor is used to measure current, which is consumed by the motor, and it is used for control of the torque of a motor. The motor torque can be changed between 0 to $106 \mathrm{~kg} \mathrm{~cm}$. The motor is equipped with a current sensor.

A voltage sensor is used to measure voltage of the motor in order to prevent over-voltage. The voltage sensor is embedded in the motor.

A temperature sensor is used to measure temperature of motor in order to prevent over-temperature. The temperature sensor is embedded in the motor. 
Ultrasonic distance sensor is low-cost distance measuring sensor. OMNIMO is equipped with four sharp ultrasonic distance sensors with a range of $4-30 \mathrm{~cm}$.

A limit switch is used to check limits. Two limit switches are used in the linear axis of OMNIMO.

\subsection{Communication units}

In this study, in order to accomplish communication between modules and communication between modules and computer a communication system is required. To construct wireless communication network Digi XBee Pro S2B wireless communication module has been used. It has 1600-meters range. Its general appearance can be seen in Fig. 3.

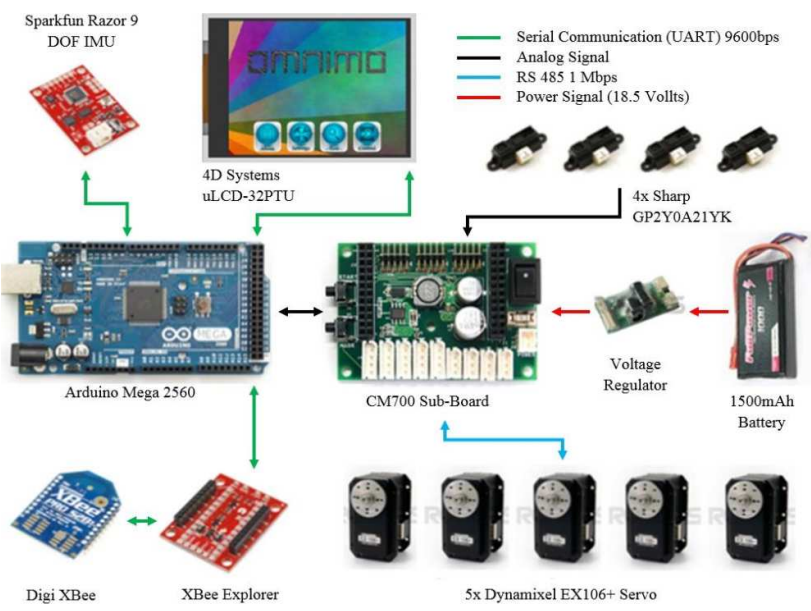

Fig. 3. Electronic system of OMNIMO.

\subsection{Regulators}

Voltage regulators are used to regulate voltage to a desired value for energizing different components. In addition, qualified regulators tolerate sudden voltage drops and prevent components from damage. OMNIMO contains three different voltage regulators, which are:

- 14.8 Volt to 3.3 Volt for IMU and XBee

- 14.8 Volt to 5 Volt for screen and ultrasonic sensor

- 14.8 Volt to 12 Volt for main controller

\subsection{Battery}

To supply power requirements of a robot there are three different ways; one of them is a power cord, the second one is batteries and the third one is producing the robot's own power with an internal combustion engine. In order to make an independent robotic system, batteries are the easy and the clean choice. For that reason, in OMNIMO project, Turnigy 18.5 Volt, 45C, $1500 \mathrm{mAh}$, high current rate lithium-polymer battery has been used. With this capacity, OMNIMO can work between 30 to 60 minutes.

\subsection{User interface units}

The robot is equipped with user interface units, making a connection between user and the robot. OMNIMO can be commanded and initiated in three different ways. First way is using a computer via wireless communication. Second way is using another module and the third way is assembling an onboard touch screen. The wireless communication system of the robot was explained before. The touch screen of OMNIMO is $4 \mathrm{D}$ systems 3.2-inch uLCD-32PTU display module with resistive touch. It is operated with 5 Volts, it has its own Atmega 8 microcontroller and it has an onboard speaker. By using the speaker, it is possible to get voiced warnings from the robot. The main purpose of putting the screen on the robot is not only commanding and initiating the robot, but also error monitoring and bug removing, during the software development.

\subsection{Complementary components}

All electromechanical systems need some electronic complementary components, such as wires, ferromagnetic or other type passive filters, signal or protocol converters, connectors and buttons. These products are indispensable for proper running electronic systems of the robot. Constructing electronic system of the robot requires precise and careful reading of datasheets. Especially, working voltage range, current needs, and proper communication protocols must be found out. Electronic system connection and communication signals can be seen in Fig. 3 and placement of electronic components inside the robot can be seen in Fig. 4 .

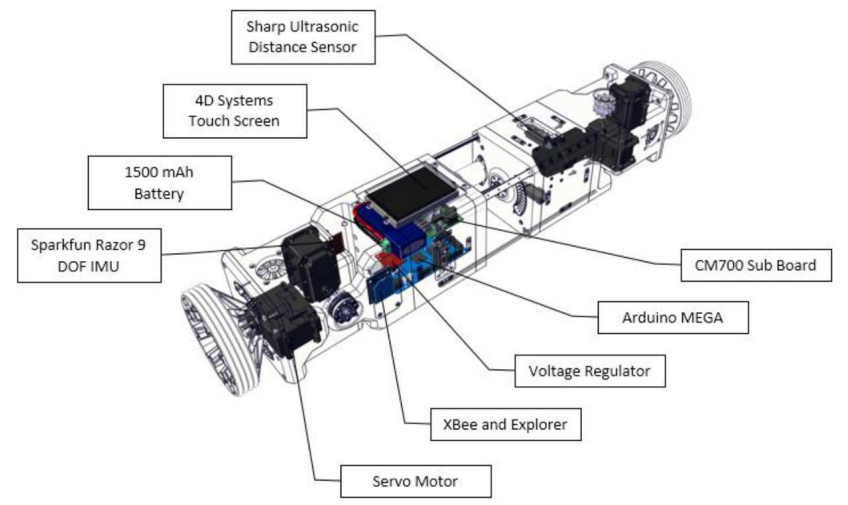

Fig. 4. Placement of electronic components inside the robot.

\section{Software}

Robot software is a set of algorithms that produce commands according to the desired tasks to control electronic and mechanical systems of the robot. Generally, commercial and traditional robots have a computer based controller and software [30,31], whereas, mobile robots have embedded controllers and software. In the OMNIMO project, a completely embedded control system 
is used, and the embedded control software and software configurations of OMNIMO are presented in this chapter. Robot software is generally developed to perform autonomous duties or tele-operated tasks. Software developed for OMNIMO supervises;

- Motion control

- Path planning

- Mission or task planning

- Internal communication between electronic robot parts

- External communication between robots or supervised controllers

- User interface etc.

Due to the complexity of robotic software, generally, it is designed and developed in the layered architecture. The layers divide the whole complex control software into small software groups. Such practice is very effective for making the debugging operations easy and also improves software modularity. Because of these reasons, main software of our modular reconfigurable robot module OMNIMO is divided into four main layers. The relations of the software architecture layers are shown in Fig. 5. They are;

- Device driver layer,

- Platform layer,

- Algorithm layer,

- User interfaces layer.

OMNIMO SOFTWARE ARCHITECTURE LAYERS

\begin{tabular}{|c|c|c|c|}
\hline $\begin{array}{c}\text { USER } \\
\text { INTERFACE } \\
\text { LAYER }\end{array}$ & ROBOT CONTROL & $\begin{array}{l}\text { SENSOR DATA } \\
\text { DISPLAY }\end{array}$ & $\begin{array}{l}\text { CONFIGURATION } \\
\text { SETTINGS }\end{array}$ \\
\hline $\begin{array}{l}\text { ALGORITHM } \\
\text { LAYER }\end{array}$ & PATH PLANNING & $\begin{array}{l}\text { OBSTACLE } \\
\text { AVOIDANCE }\end{array}$ & LOCALIZATION \\
\hline $\begin{array}{l}\text { PLATFORM } \\
\text { LAYER }\end{array}$ & LIMITATIONS & SENSOR FUSION & KINEMATICS \\
\hline $\begin{array}{r}\text { DRIVER } \\
\text { LAYER }\end{array}$ & $\begin{array}{l}\text { SENSOR } \\
\text { INTERFACE }\end{array}$ & $\begin{array}{l}\text { ACTUATOR } \\
\text { INTERFACE }\end{array}$ & $\begin{array}{c}\text { OTHER } \\
\text { INTERFACES }\end{array}$ \\
\hline
\end{tabular}

Fig. 5. Software architecture layers of OMNIMO.

\subsection{Device driver layer}

This layer is developed to handle low-level driver functions, which are required to operate sensors, actuators and communication elements. In this level, raw sensor data are turned into meaningful engineering units, as well as meaningful engineering units are turned into robot actions. In this study, for the sake of reducing complexity and getting interaction with the hardware, five different libraries are constructed for five different hardware elements. These are;

- Dynamixel EX106+ UART communication library,

- Sharp ultrasonic distance sensor calibration library,

- Sparkfun Razor 9 DOF IMU communication and calibration library,

- XBee communication library,

- Color touch screen communication library.

\subsection{Platform layer}

Platform layer is an interconnection between high-level algorithm and the drivers of the robot. This level contains physical hardware configurations, physical relations, kinematics and settings, such as motor limits and sensor thresholds. It takes over communication between elements of the robot. Translation between high-level code and low-level code is accomplished at this level. Platform layer of OMNIMO includes;

- Kinematics,

- Sensor fusion,

- Limitations.

\subsection{Algorithm layer}

Algorithm layer represents the high-level control algorithms for the robotic system. In other words, decisionmaking algorithms of the robot work on this level. All meaningful sensory information comes into this level according to the proper algorithm; such as steering, obstacle avoidance and robot locomotion strategies, which are also coded in this level. Reconfiguration and modularity are also defined at this level. OMNIMO can realize five different robots. Three of them are mobile robots and the rest are static robotic arm type robots. For locomotion of mobile robot types and static robot configurations, movement strategies are stored in this level. Also, multi module reconfiguration and combining strategies and the algorithms will be held at this level.

\subsection{User interface layer}

This layer of the algorithm provides physical interaction between the operator and the robot. This interface layer can be used for code developing, debugging and for monitoring the errors. In order to develop the main algorithm of OMNIMO, four different user interfaces or platforms have been used. They are;

- Dynamixel wizard,

- Matlab,

- SimMechanics interface,

- Touch screen interface (embedded control). 


\section{Conclusions}

The main motivation of building OMNIMO, shown in Fig. 1, was to develop an open source, completely standalone, modular reconfigurable robot module with high dexterity and flexibility for multitasking. For this reason, robot module is equipped with extensive sophisticated hardware and powerful software, to control completely new kinematic configuration, especially for modular reconfigurable robotic field, because total performance of the robotic systems is directly dependent on its hardware and software capabilities.

This paper focuses on electronic system and software architecture of modular reconfigurable robot module OMNIMO. Selected electronic components with features and reason for selection are specified. In software part, in order to reduce complexity of robotic software, control algorithms of our robot module are divided in four different layers. During the code development process, layered software can be expanded easily. By the help of the developed software and hardware integration, the desired robot architecture can be obtained and different ideas can be tested on the different robot types, which can be constructed from multiple OMNIMO modules.

\section{Acknowledgments}

This study has been supported and funded by the University of Gaziantep, Scientific Research Projects Commission under the project number MF.11.14

\section{References}

[1] A. Kılıç, S. Kapucu, J. Faculty Engin. Architect. Gazi Univ. 31, 521 (2016).

[2] T. Fukuda, S. Nakagawa, Y. Kawauchi, M. Buss, in: Proc. IEEE Int. Conf. on Robotics and Automation, 1989, p. 695.

[3] T. Fukuda, S. Nakagawa, in: Proc. IEEE Int. Conf. on Robotics and Automation, 1988, p. 1581.

[4] M. Yim, Ph.D. Thesis, Stanford University, 1994.

[5] Chirikjian, S. Gregory, in: Proc. IEEE Conf. on Robotics and Automation, 1994, p. 449.

[6] A. Castano, W.M. Shen, P. Will, Autonomous Robots 8, 309 (2000)

[7] M. Yim, D.G. Duff, K.D. Roufas, in: Proc. IEEE Int Conf. on Robotics and Automation, 2000, p. 514.

[8] E. Yoshida, S. Murata, H. Kurokawa, K. Tomita, S. Kokaji, in: Proc. 1998 IEEE/RSJ International Conference on Intelligent Robots and Systems, Vol. 2, 1998, p. 852.

[9] K. Kotay, D. Rus, M. Vona, C. McGray, in: Workshop on Algorithmic Foundations of Robotics, 1998, p. 376.

[10] J.W. Suh, S.B. Homans, M. Yim, in: Proc. IEEE Int. Conf. on Robotics and Automation, 2002, p. 4095.
[11] S. Murata, E. Yoshida, K. Tomita, H. Kurokawa, A. Kamimura, S. Kokaji, in: Proc. 2000 IEEE/RSJ International Conference on Intelligent Robots and Systems, 2000, Vol. 3, p. 2210.

[12] D. Rus, M. Vona, Autonomous Robots 10, 107 (2001).

[13] C. Ünsal, H. Kiliççöte, P.K. Khosla, Autonomous Robots 10, 23 (2001)

[14] A. Kamimura, H. Kurokawa, E. Yoshida, K. Tomita, S. Kokaji, S. Murata, in Proc. 1998 IEEE/RSJ International Conference on Intelligent Robots and Systems, Vol. 3, 2004, p. 2370.

[15] F. Mondada, G.C. Pettinaro, A. Guignard, I.W. Kwee, D. Floreano, J. L. Deneubourg, M. Dorigo, Autonomous robots 17, 193 (2004).

[16] E.H. Řstergaard, K. Kassow, R. Beck, H.H. Lund, Autonomous Robots 21, 165 (2006).

[17] S.C. Goldstein, J.D. Campbell, T.C. Mowry, Programmable Matter. Computer 38, 99 (2005).

[18] V. Zykov, W. Phelps, N. Lassabe, H. Lipson, Molecubes extended: Diversifying capabilities of open-source modular robotics, in: IROS-2008 Self-Reconfigurable Robotics Workshop, 2008.

[19] H. Kurokawa, K. Tomita, A. Kamimura, S. Kokaji, T. Hasuo, S. Murata, Int. J. Robotics Res. 27, 373 (2008).

[20] B. Salemi, M. Moll, W.M. Shen, in: Proc. 2006 IEEE/RSJ International Conference on Intelligent Robots and Systems, 2006, p. 3636.

[21] R. Moeckel, C. Jaquier, K. Drapel, E. Dittrich, A. Upegui, A.J. Ijspeert, Industrial Robot: Int. J. 33, 285 (2006).

[22] J. Gonzalez-Gomez, E. Boemo, Motion of minimal configurations of a modular robot: sinusoidal, lateral rolling and lateral shift in: Climbing and Walking Robots, Ed. M.O. Tokhi, Springer, Berlin Heidelberg 2006, p. 667.

[23] K. Gilpin, K. Kotay, D. Rus, I. Vasilescu, Int. J. Robotics Res. 27, 345 (2008)

[24] A. Sproewitz, A. Billard, P. Dillenbourg, A.J. Ijspeert, in: Proc. IEEE Int. Conf. on Robotics and Automation, 2009, p. 4259

[25] H. Wei, Y. Chen, J. Tan, T. Wang, IEEE/ASME Trans. Mechatron. 16, 745 (2011).

[26] J. Davey, N. Kwok, M. Yim, in: Proc. 2012 IEEE/RSJ International Conference on Intelligent Robots and Systems, 2012, p. 4464.

[27] A. Kılıç, S. Kapucu, Int. J. Systems Applicat. Engin. Develop. 10, 274 (2016).

[28] Robotis e-Manual v1 2\%.00, http://support.robotis.com/en/.

[29] 8-bit Atmel Microcontroller with 16/32/64KB InSystem Programmable Flash.

[30] N.G. Adar, R. Kozan, Acta Phys. Pol. A 130, 269 (2016).

[31] N.G. Adar, A.E. Tiryaki, R. Kozan, Acta Phys. Pol. A 128, B-348 (2015). 